\title{
Advances in Myiasis Treatment
}

\section{Nigam $Y^{*}$}

Biomedical Science, Swansea University, United Kingdom

\begin{abstract}
The maggots of the green bottle fly, Lucilia Sericata, have been crawling around the world for about two hundred million years. Following the evolution of man, a relationship developed between these maggots and the wounds of man. An acceptable sort of myiasis was born. In the last decade, the level of evidence recording successful outcomes of clinically applied, artificially induced myiasis on wounds using this medicinal maggot, has expanded greatly. And as modern and advanced technology helps science to unlock more doors, we are able to gain a clearer picture of the molecules and biochemical pathways by which maggots exert their effects; studies which hopefully will enrich our understanding of the clinical effects observed. The following commentary précises such new developments and summarises our current thinking on maggot / larval therapy.
\end{abstract}

Keywords: Maggots; Lucilia sericata; Myiasis; Maggot therapy; Larval therapy; Wound healing

\section{Introduction}

Maggot therapy, the use of sterile maggots to clear and cleanse necrotic wounds, was flourishing in the 1930s and early 1940s, being widely used in hospitals in the USA and Canada. Then, one day in London, a young Scottish scientist, Alexander Fleming, accidently discovered penicillin growing in his petri dishes, and by the mid-40s, the industrial antibiotic era had commenced in earnest. This marvel saw an end to maggot therapy, albeit only temporarily, while everybody excitedly began using antibiotics for everything. Overuse of antibiotics has now resulted in the emergence of several species of bacteria resistant to almost any antibiotic therapy. Seventy years later, we can no longer rely on antibiotics to treat necrotic tissue wound infections. Meanwhile, diseases, such as obesity and diabetes, previously unknown in past populations, are escalating alarmingly. Resulting from these conditions, we see a huge rise in patients with non-healing and chronic wounds, coupled with the perturbing presence of resistant microbes. As such, maggot therapy is being re-visited as an alternative to antibiotic therapy for infected tissues and is gaining renewed interest and advocacy for debridement and ultimate healing of necrotic tissues too.

\section{Wounds and Myiasis}

Maggot therapy is essentially an artificially induced myiasis. Trained clinicians apply sterile larvae of the green bottle fly (Lucilia sericata), onto a wound in serious need of debridement and often disinfection. The larvae feast on dead necrotic tissue, eliminate infection and appear to promote healthy tissue formation. After about 4 days, the larvae are removed and the wound re-assessed for further therapy. Whilst the culture of disgust and disdain that surrounds the application of maggots onto one's own body may exist, there appears nonetheless to have been a general worldwide increase in the clinical use of maggots to manage wounds. This revival, in part, is due to the numerous published reports of successful clinical outcomes following larval treatment. In addition, the last decade has seen significant technological advances that have aided our understanding of the scientific principles and physiological pathways which underlie the action of maggots in wounds, and, it is argued, that the efficiency of medicinal maggots is now beyond doubt.

Maggots appear to be able to combat wounds in three ways: debridement, disinfection and the acceleration of healing. The advances in our understanding of clinical myiasis and its treatment, therefore, stem from both clinical reports and the results of laboratory investigations, in these three arenas.

\section{Advances in medicinal maggot-led wound debridement}

This facet of maggot action is the best understood of all. It has been firmly established that maggots produce a combination of proteolytic and other digestive enzymes which are released externally in their secretions, and which get distributed over the wound as the maggot crawls around. Two key enzymes, trypsin and chymotrypsin, have been identified and studies indicate that the success of maggot enzymatic debridement is mastered through the ability of these enzymes to withstand endogenous wound inhibitors, which would normally degrade and destroy other, perhaps autolytic, enzymes [1]. Recently, larval chymotrypsin was shown to effectively degrade macromolecules present in venous leg ulcer slough, molecules such as fibrin and fibrinogen [2]. These constituents often act as key substrate sites for notorious wound bacterial biofilm to attach and accumulate. So, maggot debriding enzymes may play more than one role and indirectly and advertently help in the control or elimination of more troublesome wound microbial challenges.

Despite an increasing abundance of successful reports of maggot therapy from clinical case studies, controlled and retrospective studies, the faith in maggots as expedient wound debriders was also put to the test in the last few years through the gold standard for comparative scientific rigor - the Randomised Controlled Trial (RCT). In all RCTs undertaken and published to date however, maggot therapy has been proven to be a much more effective debriding tool that its control counterparts [3].

\section{Advances in wound disinfection by medicinal maggots}

Researchers have long been convinced that larval secretions contain effective antibacterial agents. In recent years, more and more evidence has gathered to confirm the presence of discrete antibacterial moieties

*Corresponding author: Nigam Y, Associate Professor Biomedical Science Leader of Swansea Maggot Research Group, Room 215, Vivian Tower, College of Human and Health Science, Swansea University, Singleton Park, Swansea Wales, SA2 8PP, Tel:+44(0)1792 518565; E-mail: y.nigam@swansea.ac.uk

Received February 09, 2016; Accepted February 25, 2016; Published March 03, 2016

Citation: Nigam Y (2016) Advances in Myiasis Treatment. Health Care: Current Reviews 4: 161. doi: 10.4172/2375-4273.1000161

Copyright: () 2016 Nigam Y. This is an open-access article distributed under the terms of the Creative Commons Attribution License, which permits unrestricted use, distribution, and reproduction in any medium, provided the original author and source are credited. 
within larval secretions. Modern and advanced scientific technologies have further unlocked our understanding of these secreted factors, and have shed new light on their nature. For example, a large (high molecular weight) peptide, Lucifensin, has been purified from larval secretions and various larval tissues [4]. Lucifensin has been shown to be potently active against several bacteria including $S$. pyogenes and $S$. pneumoniae. Additionally, a small antibacterial molecule, Seraticin, has been isolated in fractions collected from sterile larval secretions, with powerful activity against 12 out of 15 tested clinical isolates of MRSA, as well as a range of other pathogens. A further investigation on antimicrobial activities of compounds released externally by $L$. sericata has confirmed the presence of a range of structurally diverse compounds, and most recently, research scientists in China have reported the isolation and purification of yet another antibacterial molecule from larval secretions [5]. This protein, MAMP, has a significant inhibitory effect on $S$. aureus and appears to work by disrupting the bacterial cell membrane [6]. In addition, another group of researchers have posited that over 45 antimicrobial peptides including lucifensin, lucimycin, attacins, cecropins, diptericins, proline-rich peptides, and sarcotoxins, are coded in L. sericata. Some of these peptides possess a broad spectrum activity against microbial pathogens, including Pseudomonas aeruginosa, Proteus vulgaris, and Enterococcus faecalis, suggesting that there may be a repertoire of available antibacterial agents at the disposal of the medicinal maggot.

The inducible nature of larval antibacterial activity had been reported previously, but has also been verified yet again more recently, with results indicating that that infected larvae have much better antibacterial capacities than sterile larvae [7]. Researchers argue that the clinical wound situation would enable larvae in the infected environment to influence production of their antibacterial activities. This discovery suggests that sterile larvae placed in an infected wound, are triggered by the presence, or by oral ingestion, of surrounding bacteria, and commence production and secretion of their antibacterial agents, acting like small, antibiotic-secreting factories, and indeed, this has led some researchers to hypothesise that microbe stimulated maggots could become the next targeted natural antibiotic family [8].

In terms of the microbial burden of a wound, it is generally agreed that wound chronicity is aggravated by the presence of a biofilm. Wound biofilm is extremely difficult to eradicate; many topical treatments are ineffective, and many antibiotics, designed to attack free (planktonic) bacterial cells cannot penetrate this walled community. It has been shown that larval secretions can not only disrupt established biofilm, but can also prevent its formation. Furthermore, it was recently shown that chymotrypsin derived from larval secretions was the molecule responsible for the disruption of protein-dependent bacterial biofilm formation [9]. This detrimental effect on biofilm was observed for both nascent and pre-formed biofilms of Staphylococcus epidermidis 5179-R1.

In addition, larval secretions reportedly contain an enzyme, a DNAse, which is able to break down DNA from wound slough and is also able to digest bacterial DNA [10]. This discovery is significant since extracellular DNA is an important requirement for some bacterial species to instigate biofilm formation. If DNAse present in larval secretions eliminates all sources of extracellular DNA, then this may explain the observed anti-biofilm effects of larvae and their secretions.

Interestingly, like the antibacterial molecules previously mentioned, larval molecules associated with disruption of biofilm have also been shown to be inducible, for example, secretions collected from larvae incubated with bacteria were able to better destroy P. aeruginosa biofilm [11] Other studies have considered the effects of larvae on biofilm production upon surfaces commonly used in a medical setting, and a recent study found that larval secretions were able to both prevent biofilm formation, and disrupt existing biofilms of P. aeruginosa [12]. The study, which encompassed many different bacterial species, noted reduced biofilm formations after treatment with larval secretions on polyethylene, titanium and stainless steel surfaces, with a maximal reduction in biofilm formation of $92 \%$. This result may be of great significance, especially when considering peri-prosthetic infections. It remains to be seen if anti-biofilm components from maggot secretions can be exploited by the disciplines of nanotechnology and bioengineering, and utilised to produce prostheses capable of combatting such infections.

It has also been newly revealed that $L$. sericata is capable of producing and externalising antifungal components, and of particular note, is the secretion of a small molecule $(<500 \mathrm{Da})$, anti-Candida factor [13]. It seems highly probable therefore, that in a wound, larvae secrete distinct factors which are able to combat wound pathogens, as well as interfering with bacterial biofilm formation and establishment.

\section{Acceleration of wound healing by medicinal maggots}

Of all the advances in myiasis treatment, the understanding behind the action of maggot therapy and its role in acceleration of new tissue growth is possibly the most exciting. While progress in our pre-clinical and scientific knowledge gathers momentum, it has to be said that recent RCTs which have assessed wound closure and healing, have not found any significant difference between larval therapy and other therapies. Nonetheless, numerous clinical studies have been performed which show that the application of maggot therapy exhibited a significantly shorter time to granulation, and to overall healing of lesions. For example, Marineau et al. [14] examined complex diabetic foot wounds in a set of 23 patients and found that $74 \%$ of them showed enhanced granulation tissue formation. The presence of poorly perfused tissue in the impairment of wound healing has always been a clinical obstacle. An interesting report was recently published by a team of Japanese surgeons, who measured an increase in skin perfusion pressure surrounding a post-amputation chronic wound, after MT on a patient with critical limb ischemia. Following MT, skin perfusion pressure increased dramatically, from 12 to $54 \mathrm{mmHg}$ on the dorsal aspect of the foot, and from 17 to $44 \mathrm{mmHg}$ on the plantar aspect. The authors surmised that somehow the presence of maggots on the wound had contributed to the increase in blood supply to the ischaemic wound, providing more clinical evidence that healing may be actively accelerated following a dose of maggots [15].

Not to be outdone by such fascinating clinical findings, laboratory science too has investigated the effects of maggot secretions on key cells and molecules known to be pivotal to wound healing. Such investigations have led to discoveries which begin to reveal the actual processes and molecular mechanisms that may play a part a role in wound healing. For example, several studies suggest that the beneficial effects of maggots may lie in their ability to reduce levels of pro-inflammatory factors thereby inhibiting on-going inflammation. Yet other researchers have shown that maggot secretions are capable of breaking down key components of the Complement Cascade, a system of potent serum enzymes which can trigger an inflammatory response [16].

Central to wound healing is the migration of essential cells such as dermal cells, fibroblasts and microvascular cells into the wound bed. Recently, Wang et al. [17], showed a significant increase in the migration of human microvascular epidermal cells following exposure to maggot secretions. Using wound healing assays to examine the possibility that 
there may be distinct pathways activated, the investigators found that the migration was indeed due to the activation of a key signalling protein AKT1, which normally regulates cell migration during wound healing.

Fibroblasts are the fundamental cells responsible for the production of collagen and ECM, and therefore healthy granulation tissue. Maggot secretions have previously been reported to enhance fibroplasia, and, recently, other researchers have added evidence to support enhanced fibroblast proliferation. Polakovicova et al. [18] showed that when fibroblasts were cultured with extracts from maggot salivary glands, the cells responded with an increased metabolism and protein production, and the formation of microfibrillar nets, structures which are requisite for fibroblast cell migration.

Additionally, many researchers have shown that maggot secretions and larval therapy can up-regulate the production of growth factors, the body's own protein molecules which have a huge role in the wound healing process, helping with the stimulation and proliferation of new tissue. Honda et al. [19] recently reported that blood samples from patients treated with maggots showed a remarkable increase in endogenous HGF, after just a single application. The authors credited the increased levels of HGF to the promotion of healthy granulation tissue. In pre-clinical investigations, it was found that in the presence of maggot secretions, macrophages significantly increased their production of pro-angiogenic $\beta F G F$ and VEGF [20], and other work, too, supports the notion of maggot secretions promoting angiogenesis. The amino acids L-histidine, 3-guanidinopropionic acid (GPA) and L-valinol were identified in maggot secretions, and it was demonstrated that these isolated components specifically enhanced the proliferation of human vascular endothelial cells, whilst having no effect on fibroblasts, with the amino acid valinol eliciting the greatest increase in endothelial cell proliferation [21].

So, in conclusion, it appears that in maggot therapy may have some beneficial and practical aspects. Maggots are able to digest necrotic debris from an unhealthy wound, disinfect and cleanse at source, and most likely promote the formation and development of new healthy tissue. These advances in our understanding have been possible because of the tireless efforts of both clinicians and laboratory scientists, which have provided us with a much better apprehension of the wound healing potential of medicinal maggot therapy.

\section{References}

1. Telford G, Brown AP, Kind A, English JS, Pritchard DI (2010) Maggot chymotrypsin 1 from Lucilia sericata is resistant to endogenous wound protease inhibitors. Br J Dermatol 164: 192-196.

2. Pritchard DI, Brown AP (2013) Degradation of MSCRAMM target macromolecules in VLU slough by Lucilia sericata chymotrypsin 1 (ISP) persists in the presence of tissue gelatinase activity. Int Wound $\mathrm{J}$.

3. Sun X, Jiang K, Chen J, Wu L, Lu H, et al. (2014). A systematic review of maggot debridement therapy for chronically infected wounds and ulcers. International Journal of Infectious Diseases 25: 32-37.

4. Cerovsky V, Slaninova J, Fucik V, Monincova L, Bednarova L, et al. (2011) Lucifensin, a novel insect defensin of medicinal maggots: synthesis and structural study. Chembiochem 12: 1352-61.

5. Kruglikova AA, Chernysh SI (2011)Antimicrobial compounds from the excretions of surgical maggots, Lucilia sericata (Meigen) (Diptera, Calliphoridae). Entomol Rev 91: 813-819.
6. Zhang Z, Wang J, Zhang B, Liu H, Song W, et al. (2013) Activity of antimicrobial protein from maggots against Staphylococcus aureus in vitro and in vivo. Int $\mathrm{J}$ Mol Med 31:1159-1165.

7. Kawabata T, Mitsui H, Yokota K, Ishino K, Oguma K, et al. (2010) Induction of antibacterial activity in larvae of the blowfly Lucilia sericata by an infected environment. Med Vet Entomol 24: 375-381.

8. Wang XY, Li XR, Gao L, Wang JN (2014) Could microbe stimulated maggots become a targeted natural antibiotics family? Med Hypotheses 83:60-61.

9. Harris LG, Nigam Y, Sawyer J, Mack D, Pritchard DI (2013) Lucilia sericata chymotrypsin disrupts protein adhesin-mediated staphylococcal biofilm formation. Appl Environ Microbiol 79: 1393-1395

10. Brown A, Horobin A, Blount DG, Hill PJ, English J, et al. (2012) Blow fly Lucilia sericata nuclease digests DNA associated with wound slough / eschar and with Pseudomonas aeruginosa biofilm. Med Vet Entomol 26:432-439.

11. Jiang KC1, Sun XJ, Wang W, Liu L, Cai Y, et al. (2012) Excretions / Secretions from Bacteria-Pretreated Maggot Are More Effective against Pseudomonas aeruginosa Biofilms. PLoS ONE 7: e49815.

12. Cazander G, van de Veerdonk MC, Vandenbroucke-Grauls CM (2010) Maggot excretions inhibit biofilm formation on biomaterials. Clin Orthop Relat Res 468: 2789-96.

13. Evans R, Dudley E, Nigam Y (2015) Detection and partial characterisation of antifungal bioactivity from the secretions of the medicinal maggot, Lucilia sericata. Wound Repair Regen 3: 361-368.

14. Marineau ML, Herrington MT, Swenor KM, Eron LJ (2011) Maggot debridement therapy in the treatment of complex diabetic wounds. Hawaii Med J 70: 121124

15. Maeda TM, Kimura CK, Takahashi KT, Ichimura KI (2014) Increase in skin perfusion pressure after maggot debridement therapy for critical limb ischaemia. Clin Exp Dermatol 39: 911-914

16. Cazander G, Schreurs MW, Renwarin L, Dorresteijn C, Hamann D, et al. (2012) Maggot excretions affect the human complement system. Wound Repair Regen 20: 879-886.

17. Wang SY, Wang K, Xin Y, Lv DC (2010) Maggot excretions / secretions induces human microvascular endothelial cell migration through AKT1. Mol Biol Rep 37: $2719-2725$.

18. Polakovicova S, Polak S, Kuniakova M, Cambal M, Majtan J, et al. (2011) The effect of salivary gland extract of Lucillia sericata maggots on the human derma fibroblasts proliferation. OnLine J. Biol. Sci., 11: 96-100.

19. Honda K, Okamoto K, Mochida Y, Ishioka K, Oka M, et al (2011) A nove mechanism in maggot debridement therapy: protease in excretion / secretion promotes hepatocyte growth factor production. Am J Physiol Cell Physiol 301 : C1423-C1430

20. van der Plas MJ, van Dissel JT, Nibbering PH (2009) Maggot secretions skew monocyte-macrophage differentiation away from a pro-inflammatory to a proangiogenic type. PLoS ONE 4: e8071.

21. Bexfield A, Bond AE, Morgan, Wagstaff J, Newton RP, et al. (2010) Amino acid derivatives from Lucilia sericata excretions / secretions may contribute to the beneficial effects of maggot therapy via increased angiogenesis. $\mathrm{Br} \mathrm{J}$ Dermato 162: 554-562. 